Gárgano, S. (2020). Análisis de las estrategias de evaluación de los aprendizajes de las materias de Fisiología Humana y Aplicada de las Carreras de Educación Física. Revista Electrónica Interuniversitaria de Formación del Profesorado, 23(3), 201-215.

DOI: https://doi.org/10.6018/reifop.366371

\title{
Análisis de las estrategias de evaluación de los aprendizajes de las materias de Fisiología Humana y Aplicada de las Carreras de Educación Física
}

\author{
Sofía Gárgano \\ Instituto de Investigaciones en Humanidades y Ciencias Sociales (IdIHCS) - Consejo \\ Nacional de Investigaciones Científicas y Técnicas (CONICET), Universidad Nacional de La \\ Plata (UNLP). Comisión de Investigaciones Científicas de la Provincia de Buenos Aires (CIC).
}

\section{Resumen}

Ante las nuevas demandas del campo educativo en torno a la enseñanza y el aprendizaje, este estudio tiene por objetivo comprender la valoración de los docentes y los estudiantes sobre las estrategias de evaluación de los aprendizajes utilizadas en las materias vinculadas a la salud de las Carreras de Educación Física de la FaHCE-UNLP, éstas son Fisiología Humana y Fisiología Aplicada. La metodología es mixta, diseño de triangulación concurrente. Para la recolección de datos se utilizaron entrevistas y cuestionarios. Los resultados fueron analizados mediante los programas SPSS y Atlas.ti. La experiencia de estudiantes y docentes indica que la evaluación debería ser formativa, orientada hacia el aprendizaje y, por tanto, continua, formando parte de todo el proceso de aprendizaje y valorándose diferentes tipos de aprendizajes y competencias, útiles para la futura práctica profesional y para la vida personal.

\section{Palabras clave}

Estrategias de evaluación; salud; fisiología; Educación Física.

\section{Contacto:}

Sofía Gárgano.gargano.sofia@gmail.com

El artículo presenta resultados fruto del proyecto de investigación derivado del trabajo de tesis doctoral para optar el grado de Doctora en Ciencias de la Educación, FaHCE-UNLP. La tesis fue entregada en mayo de 2019. 


\title{
Analysis of the evaluation strategies of the learning of the subjects of Human and Applied Physiology of the Careers of Physical Education
}

\begin{abstract}
Given the new demands of the educational field regarding teaching and learning, this study aims to understand the assessment of teachers and students on the evaluation strategies of learning used in the subjects related to the health of the Careers of Physical Education of the FaHCE-UNLP, these are Human Physiology and Applied Physiology. The methodology is mixed, concurrent triangulation design. Interviews and questionnaires were used for data collection. The results were analyzed using the SPSS and Atlas.ti programs. The experience of students and teachers indicates that the evaluation should be formative, oriented towards learning and, therefore, continuous, being part of the whole learning process and valuing different types of learning and skills, useful for future professional practice and for personal life.
\end{abstract}

\section{Key words}

Assessment strategies; Health; physiology; Physical education.

\section{Introducción}

Este estudio se desprende de una investigación más amplia que tuvo por objetivo comprender la valoración de los docentes y los estudiantes en base a sus experiencias, sobre la enseñanza de las materias vinculadas a la salud en las Carreras de Licenciatura y Profesorado en Educación Física (CEF) de la Facultad de Humanidades y Ciencias de la Educación (FaHCE) de la Universidad Nacional de La Plata (UNLP), con el fin de vislumbrar necesidades y preferencias acerca de la enseñanza y el aprendizaje en esta área específica. Para ello, las unidades o categorías de análisis utilizadas fueron las estrategias de enseñanza, las características para ser un buen docente y las estrategias de evaluación de los aprendizajes. Este estudio se centra en las estrategias de evaluación de los aprendizajes.

\section{Estudiar sobre docencia universitaria}

La pertinencia social para investigar sobre docencia universitaria, está en relación al paradigma educativo vigente. Los paradigmas del siglo pasado, han perdido vigencia histórica; en la actualidad, la pertinencia social está en función de la información, de la comunicación y de la sociedad del conocimiento, por lo que el aprendizaje debe ser continuo y tener la finalidad de formar a las personas para que éstas puedan construir su propio conocimiento (Colina Colina, 2007).

\section{La evaluación de los aprendizajes}

La evaluación puede definirse, de forma general, como un proceso que se basa en obtener información y analizarla, para luego formular un juicio (Fraile Aranda, 2009). También, puede definirse como un proceso continuo e íntimamente integrado a la enseñanza que aporta 
distintas informaciones y cumple diversas funciones (Davini, 2008). Existen diferentes perspectivas acerca de cómo evaluar los aprendizajes, principalmente, se pueden identificar formas de evaluar desde una mirada tradicional, y modos de evaluación que intentan ser superadores de la evaluación tradicional, denominados, evaluación formativa y/o evaluación orientada hacia el aprendizaje.

Desde una mirada tradicional, la evaluación tiene por objetivo calificar para comprobar los aprendizajes adquiridos, al final del proceso de aprendizaje. El medio que se utiliza para calificar es el examen, siendo generalmente, escrito u oral. La evaluación se caracteriza por ser técnica, objetiva y eficaz, donde el estudiante ocupa un rol pasivo en todo lo que a ella respecta (López Pastor, 2006; Castejón Oliva, López-Pastor, Julián Clemente y Zaragoza Casterad, 2011).

Este tipo de evaluación al final del ciclo, mediante pruebas escritas u orales, es criticada por la literatura pedagógica, ya que suele generar frustración y desmotivación en los estudiantes, y promueve que éstos estudien de memoria o aprendan según la forma en que serán evaluados. Por ello, la evaluación no debería limitarse al final del ciclo de enseñanza y habría que suprimir la idea de que los buenos estudiantes son los que obtienen mayor puntaje en las pruebas (Davini, 2008). Además, podría ocurrir que, si un gran porcentaje de sus estudiantes aprueba el examen, el docente se sienta satisfecho con su labor, pero no puede saberse con precisión si aquello es evidencia del aprendizaje de los estudiantes o se debe a que quizá la prueba consistió en repetir de memoria y sin analizar, la información expuesta en las clases. Por el contrario, si hubiera mayor cantidad de desaprobados, el docente podría justificar tal situación aludiendo a la falta de estudio de los estudiantes o, en menor medida, a alguna cuestión pedagógica, lo que lo llevaría a reflexionar sobre el proceso de enseñanza y aprendizaje (Córdoba Gómez, 2006).

Las pruebas escritas u orales deberían complementarse con diferentes tipos de actividades a lo largo del ciclo de enseñanza y aprendizajes, además de tener por objetivo ayudar a aprender, en lugar de limitarse a calificar. Cualquier tipo de evaluación es un aprendizaje en sí mismo, ya que a partir de ésta, los estudiantes pueden, por ejemplo, reflexionar acerca de su falta de estudio en tal o cual tema, lo que significa que la evaluación aporta información sobre el aprendizaje alcanzado al momento. Dicho aporte, es la potencialidad de la evaluación para mejorar el aprendizaje. Además, el diseño del examen, es decir, la forma en que los estudiantes serán evaluados, influye en cómo éstos estudian, ya que no es lo mismo prepararse para una prueba escrita u oral en la que se deban describir conceptos, que para una prueba de iguales características, en la que haya que realizar diferentes tareas, como son planificar, elaborar, deducir, interpretar, evaluar, etc.; en la primera prueba se potenciará, principalmente, la memorización de conceptos, mientras que a través de la segunda, se promoverá el despliegue de diferentes habilidades (Padilla Carmona y Gil Flores, 2008).

Como propuestas superadoras de la evaluación tradicional, los tipos de evaluación denominados formativos u orientados hacia el aprendizaje, son los que prevalecen en la bibliografía científica. Se hable de evaluación formativa o de evaluación orientada hacia el aprendizaje, su implementación tiene por objetivo la mejora de los aprendizajes.

La evaluación formativa es una evaluación democrática, ya que incorpora a los estudiantes como partícipes en las decisiones que se toman y busca desarrollar en los estudiantes responsabilidades y autonomía en el proceso de aprendizaje (Fraile Aranda, 2009; Castejón Oliva et al., 2011; Gómez Ruiz y Quesada Sierra, 2017). Además, la evaluación formativa es continua, ya que forma parte de todo el proceso de aprendizaje. En dicho proceso, se valoran diferentes tipos de aprendizajes y competencias sobre los que los docentes han planificado (López Pastor, 2006). Mediante la evaluación formativa, se busca adquirir aprendizajes que 
sean útiles para la futura práctica profesional y para la vida personal. Para que los aprendizajes perduren en el tiempo, las estrategias de evaluación tendrán que ser innovadoras y deberán promover la comprensión, el desarrollo de competencias y el despliegue de habilidades cognitivas (López Pastor, 2006; Hernández Pina, Arán Jara y Salmerón Pérez, 2012). Cuando se habla de competencias se hace referencia a capacidad de transferir y adaptar esquemas de actividad a distintas situaciones que se puedan presentar en la práctica profesional. Las competencias no se adquieren en un momento particular sino que se crean y modifican de forma constante de acuerdo a las necesidades y requerimientos de la práctica (Pavié, 2011).

Bajo esta misma perspectiva, los estudios que hacen referencia a la evaluación orientada hacia el aprendizaje, explican que ésta se basa en tres aspectos, ellos son, que las tareas de evaluación sean planteadas como tareas de aprendizaje, involucrar a los estudiantes en la evaluación, y que los resultados de la evaluación sean presentados haciendo un feedback con los estudiantes (Gessa Perera, 2011). Para que una evaluación se oriente hacia el aprendizaje, se deben considerar algunos aspectos como: que las tareas de evaluación formen parte de las tareas de aprendizaje; que los estudiantes actúen sobre la información recibida y la utilicen para continuar el proceso de aprendizaje; que los estudiantes se involucren evaluando sus propias tareas, aspecto considerado esencial para su futura práctica profesional. Además, para que las tareas de evaluación estimulen el aprendizaje deben implicar esfuerzo y dedicación, deben estar distribuidas en las semanas, deben ser productivas para los estudiantes y deben transmitir expectativas altas y claras a los estudiantes. Es importante que la evaluación se corresponda con los objetivos planteados en la asignatura, es decir, que las tareas de evaluación sean auténticas, en sintonía con los objetivos. Es esperable que las tareas de evaluación se diseñen con el objetivo de favorecer el desarrollo de habilidades, competencias y actitudes para la futura práctica profesional. Para implementar una evaluación orientada al aprendizaje, los docentes deben aprovechar el interés y la importancia que los estudiantes le otorgan a la evaluación. El estudiante debe participar del proceso de evaluación, ya sea en la planificación, en la ejecución o en la calificación (Padilla Carmona y Gil Flores, 2008).

En este sentido, las principales modalidades de evaluación formativa u orientada hacia el aprendizaje, son la autoevaluación, la evaluación entre iguales y la coevaluación (Gómez Ruiz y Quesada Sierra, 2017). Mediante la autoevaluación, los estudiantes deben valorar su propio trabajo. El objetivo principal de esta modalidad, es desarrollar la autonomía y la responsabilidad de los estudiantes, con el fin de que esta práctica, conduzca a que continúen evaluando su desempeño cuando sean profesionales (Fraile Aranda, 2009; Castejón Oliva et al., 2011; López Pastor, 2006). La evaluación entre iguales, hace referencia a la evaluación entre compañeros (Gómez Ruiz y Quesada Sierra, 2017). Por último, en la coevaluación, los estudiantes negocian y acuerdan con los docentes la forma en que serán evaluados, prevalece la responsabilidad compartida y el diálogo para tomar decisiones (Gómez Ruiz y Quesada Sierra, 2017). Cabe aclarar que, algunos autores, denominan coevaluación a la evaluación que realizan entre los estudiantes y, como otra modalidad, describen la evaluación compartida, definiéndola como aquella que se produce entre docentes y estudiantes (López Pastor, 2006). En este estudio se utiliza la definición otorgada por Gómez Ruiz y Quesada Sierra (2017).

Otra contribución a la evaluación formativa, es la "evaluación auténtica". Por medio de la evaluación auténtica, se intenta mejorar la evaluación a través de dos estrategias: por un lado, propone utilizar herramientas e instrumentos de evaluación que por lo general no son utilizados o se dejan en un segundo plano, por otro lado, plantea acercar la evaluación al proceso de enseñanza, evitando que ésta se limite al cierre final. El marco general de la 
evaluación auténtica es crear formas de trabajo que representen situaciones de la vida real y de esa forma poner a prueba lo que los estudiantes aprendieron, por ejemplo, realizando demostraciones reales como escribir manifestando lo que piensan, leer interpretando lo que leen, expresarse oralmente y mantener un discurso ordenado y claro, mostrar su creatividad y su capacidad de investigación y resolución de problemas, entre otros, proponer en estas evaluaciones procesos mentales más complejos y estimulantes, que la simple respuesta a cuestionarios, incorporar la evaluación de distintas capacidades y no sólo de conocimiento, por ejemplo, expresivas, creativas, prácticas y sociales, reconocer que puede existir más de un enfoque para dar una respuesta, usar criterios de evaluación apropiados y dar importancia a los productos que se desarrollen espontáneamente en la clase (Davini, 2008).

A pesar de que se ha estudiado y comprobado que una participación activa de los estudiantes en la evaluación, genera aceptación y resultados positivos, la realidad es que los estudiantes no suelen tener una participación activa en torno a lo que la evaluación respecta, siendo los docentes quienes toman las decisiones sobre qué, cómo y cuándo evaluar y calificar. Según algunos docentes, los estudiantes son inmaduros para tomar este tipo de decisiones, además de considerar a estas actividades como una forma de desperdiciar el tiempo y una pérdida en la centralidad de los contenidos. Por otra parte, los estudiantes, al estar acostumbrados a transitar su proceso de aprendizaje de forma pasiva y confortable, muchas veces, no reciben favorablemente propuestas innovadoras que los hagan participar activa y autónomamente (Castejón Oliva et al., 2011).

En este sentido el estudio de Moreno Olivos (2009), arrojó que, aunque existe un fuerte discurso sobre la importancia de implementar una evaluación formativa y continua, la evaluación que predomina es de tipo tradicional, la cual, en lugar de cumplir un rol pedagógico, es decir, orientador, constructivo y de retroalimentación para mejorar los aprendizajes, se usa como mecanismo de control, predisponiendo a los estudiantes a que tengan una postura pasiva y acrítica sobre la evaluación. El docente es el protagonista del proceso, en el que evaluar y calificar, son un fin en sí mismos, en lugar de ser un medio para aprender. En este contexto, se considera al estudiante como único responsable de su bajo rendimiento académico, lo cual se atribuye a una formación escolar deficiente, a la poca dedicación y al pobre tiempo que disponen para estudiar, entre otros, lo cual da cuenta de una falta de autocrítica por parte de los docentes. Esta situación, trae como consecuencia que los docentes no se preocupen por mejorar sus prácticas de enseñanza, por lo que no diseñan y llevan a cabo diferentes tipos de clases, donde se incorpore la participación de los estudiantes, por ejemplo, implementando la autoevaluación y la coevaluación en sus clases. Todo ello, representa un círculo negativo que no permite avanzar hacia la mejora de los aprendizajes.

Se espera que las estrategias de evaluación de los aprendizajes sean innovadoras, auténticas, dejando atrás prácticas evaluativas tradicionales, que poco promueven la comprensión y el ejercicio de habilidades cognitivas. Desde el enfoque actual, basado en el desarrollo de competencias, la evaluación será diseñada con el fin de promover habilidades de pensamiento (Hernández Pina et al., 2012), y los estudiantes tendrán la posibilidad de involucrarse en el diseño de la evaluación, negociando con el docente cómo serán evaluados (Castejón Oliva et al., 2011). Por tanto, la evaluación formativa y la evaluación orientada al aprendizaje buscan generar un aprendizaje a lo largo de toda la vida, es decir, intentan ser una evaluación sostenible, que generen los conocimientos, habilidades y actitudes que se requieren para las actividades de aprendizaje a lo largo de la vida. Para lograrlo, se preocupan por que las tareas estimulen a los estudiantes a desarrollar el pensamiento, hacer juicios complejos y tomar decisiones (Padilla Carmona y Gil Flores, 2008). 


\section{Metodología}

Para confeccionar la metodología se han tomado los aportes de Hernández Sampieri (2014) y de Hernández Sampieri, Fernández Collado y Baptista Lucio (2006).

Este estudio persigue 1) conocer cuáles estrategias de evaluación de los aprendizajes se utilizan con más frecuencia, cuáles se prefieren y cuáles se rechazan, y 2) vislumbrar por qué determinadas estrategias de evaluación de los aprendizajes son más apreciadas que otras y entender las necesidades de los estudiantes y de los docentes en base a sus experiencias como aprendices o como profesores.

Se utiliza una metodología mixta, ya que ésta permite alcanzar una perspectiva holística e integral del fenómeno, debido a que se combinan la posibilidad de medir, generalizar y ampliar información (método cuantitativo), con el acercamiento, la profundización y la comprensión de la complejidad del problema (método cualitativo).

\section{Diseño de la investigación}

Se utiliza una triangulación concurrente, ya que se busca corroborar resultados y efectuar validación cruzada entre datos cuantitativos y cualitativos, aprovechando las ventajas de cada método. De forma simultánea se recolectan y analizan los datos cuantitativos y cualitativos, y luego en la interpretación se explican los dos tipos de resultados, realizando comparaciones entre las bases de datos. Además, los resultados se presentan aportando datos cuantitativos, seguidos por las categorías cualitativas, pudiendo corroborar la validez de criterio de las pruebas. También, se toman algunos aportes del diseño anidado concurrente de varios niveles, ya que se ha obtenido información de diferentes grupos y niveles de análisis.

Aspectos cuantitativos del diseño: es descriptivo, ya que mediante la recolección de la información se busca caracterizar el fenómeno que se somete a análisis; es no experimental, ya que se observan fenómenos para luego analizarlos sin manipular variables; es de tipo transversal.

Aspectos cualitativos del diseño: se identifica con un diseño fenomenológico, ya que se busca conocer y entender las experiencias de personas ante un fenómeno o diferentes perspectivas sobre éste, por lo que, las personas seleccionadas como objetos de estudio, son aquellas que han compartido tales experiencias. El análisis de los datos consiste en reconocer unidades de significado, descripciones del fenómeno y experiencias compartidas, obteniéndose como producto de la investigación, un reporte donde se describe el fenómeno y la experiencia común de varios participantes con respecto a éste. No se busca generar un modelo a partir de las experiencias, sino que se explora, describe y comprende lo que los sujetos tienen en común, en función de sus experiencias acerca de tal fenómeno, identificándose a partir de ellas, las categorías.

\section{El muestreo}

El universo bajo estudio son los docentes y estudiantes de las CEF de la FaHCE-UNLP. La muestra se compuso por 10 docentes y un total de 378 estudiantes. Tanto desde el enfoque cuantitativo como cualitativo, la muestra puede clasificarse como no probabilística o dirigida, seleccionada de acuerdo con propósitos particulares, es de carácter homogéneo o de casos tipo, ya que se entrevistaron docentes que compartían características similares (enseñanza de la misma área temática) y se encuestaron estudiantes que hayan cursado las materias del 
área de la salud. También es una muestra por oportunidad, ya que se aprovecharon los momentos en que los estudiantes estaban en la facultad participando de alguna actividad.

El número de casos fue seleccionado en torno al entendimiento del fenómeno, es decir, en función a la saturación de categorías.

Se analizaron los programas de las asignaturas y seminarios de las CEF de la FaHCE-UNLP para determinar qué materias se hallaban vinculadas a la salud, éstas son:

Las asignaturas Fisiología Humana y Fisiología Aplicada a la Educación Física, y los seminarios de "Evaluación de la aptitud física en el campo de la salud", "Promoción y prescripción de actividad física para la salud" y "Cuerpo, salud y política". Se excluyó este último seminario ya que los fundamentos y objetivos, los contenidos y la bibliografía presentes en su programa, difieren del resto de las asignaturas y seminarios vinculados a la salud.

Instrumentos de recolección de datos: Entrevistas semiestructuradas en docentes, cuestionarios con preguntas abiertas y cuestionarios con preguntas cerradas, ambos autoadminitrados por estudiantes.

Clase de datos recolectados: Todos los instrumentos permiten una codificación numérica y de análisis como texto.

\section{Diseño del cuestionario de tipo cerrado}

Una de las formas más frecuentes de valorar las preferencias de los estudiantes acerca de la enseñanza es mediante escalas, encuestas o entrevistas semiestructuradas, pudiendo éstas, ser adaptadas según los objetivos de cada investigación particular. Para este estudio se adaptó el cuestionario denominado "Cuestionario sobre las expectativas de enseñanza de los estudiantes universitarios" diseñado por De la Fuente et al. y publicado en el año 2002 (Berbén, de la Fuente, Benítez y Fernández Cabezas, 2007), cuya versión original fue mencionada en el párrafo anterior: "University students' expectations of teaching" (USET) creado por los investigadores Sander, Stevenson, King y Coates (2000).

El "Cuestionario sobre las expectativas de enseñanza de los estudiantes universitarios", presenta una hoja de instrucciones separada en 3 secciones. De éste se tomó y adaptó la sección $B$, en la cual los estudiantes debían valorar, de una lista de opciones, los tres métodos de evaluación considerados por ellos como más efectivos: el preferido con el número 1, el siguiente elegido con el número 2, y el tercero seleccionado con el 3; las opciones fueron:

Trabajo en clase y luego participar activamente de las puestas en común; Proyectos de investigación; Exámenes; Entrega de monografías de forma individual (trabajo con fecha de entrega, domiciliario); Entrega de monografías de forma grupal; Exposiciones orales; Realización de actividades en clase con posterior entrega.

\section{Diseño del cuestionario de tipo abierto}

Se priorizaron preguntas abiertas y respuestas de forma escrita, en lugar de la oralidad que permite la entrevista, debido a que la escritura posibilita que las respuestas sean pensadas, y además, permite recabar mayor cantidad de información en menos tiempo, ya que, el cuestionario pudo ser entregado y respondido, al mismo tiempo, por diferentes estudiantes.

En el cuestionario, los estudiantes debieron colocar la fecha, e indicar si cursaron o no las asignaturas Fisiología Humana y Fisiología Aplicada a la Educación Física. Luego, para conocer 
las estrategias de evaluación de los aprendizajes valoradas por ellos, se confeccionó la siguiente pregunta a desarrollar:

En torno a estas asignaturas (Fisiología Humana y Fisiología Aplicada a la Educación Física) y teniendo en cuenta, también, seminarios sobre temas similares: ¿Cómo te gustaría que te evalúen? Describí una forma que vivenciaste y te gustó, o realizá una propuesta sobre cómo te gustaría que te evalúen.

\section{Definición de las variables}

La información fue agrupada para su análisis, en función de las respuestas de docentes y estudiantes, en las siguientes subcategorías:

Tabla 1.

Distribución de alumnos participantes por titulación

\section{Categoría de análisis Subcategorías}

\begin{tabular}{lll}
\hline Estrategias & de & -Evaluación a desarrollar e integrar \\
evaluación de & los & contenidos y examen multiple choice \\
aprendizajes & & -Evaluación que incorpore el desarrollo \\
& de habilidades prácticas \\
& -Evaluación continua \\
\hline
\end{tabular}

\section{Análisis e interpretación de los datos}

Los datos cuantitativos se analizaron mediante estadística descriptiva e inferencial, utilizando hojas de cálculo de Microsoft Excel y el programa estadístico IBM SPSS Statistics 19, y la información cualitativa a través de codificación y evaluación temática, por medio de análisis manual y del programa de análisis cualitativo, Atlas.ti, además de análisis combinados.

\section{Resultados y análisis}

A continuación se presentan los primeros hallazgos acerca de las estrategias de evaluación de los aprendizajes, y luego se describe y analiza la información obtenida, organizada en apartados correspondientes con las subcategorías.

\section{Primeros hallazgos sobre las estrategias de evaluación de los aprendizajes}

Las estrategias de evaluación de los aprendizajes, valoradas en una primera instancia por los estudiantes, son las obtenidas a partir de una lista de opciones (cuestionario cerrado) y se presentan en la siguiente tabla:

La información expuesta en la tabla demuestra que los "trabajos en clase y luego participar activamente de las puestas en común" es la estrategia de evaluación considerada como más efectiva por los estudiantes (31,7\%), en segundo lugar la "entrega de monografías de forma 
individual" (17,5\%), y el tercer lugar, por escasa diferencia entre las opciones, es para los "exámenes" (15\%).

Tabla 2.

Estrategias de evaluación de los aprendizajes, valoradas mediante el cuestionario cerrado

\begin{tabular}{|c|c|c|c|c|c|c|}
\hline \multirow[t]{2}{*}{ Estrategia de evaluación más efectiva } & \multicolumn{2}{|l|}{$1^{\circ}$} & \multicolumn{2}{|l|}{$2^{\circ}$} & \multicolumn{2}{|l|}{$3^{\circ}$} \\
\hline & $\mathbf{N}$ & $\%$ & $\mathbf{n}$ & $\%$ & $\mathbf{N}$ & $\%$ \\
\hline Entrega de monografías de forma grupal & 7 & 2,9 & 21 & 8,8 & 33 & 13,8 \\
\hline $\begin{array}{l}\text { Entrega de monografías de forma } \\
\text { individual }\end{array}$ & 31 & 12,9 & 42 & 17,5 & 33 & 13,8 \\
\hline Exámenes & 40 & 16,7 & 32 & 13,3 & 36 & 15 \\
\hline Exposiciones orales & 23 & 9,6 & 27 & 11,3 & 27 & 11,3 \\
\hline Proyectos de investigación & 32 & 13,3 & 36 & 15 & 25 & 10,4 \\
\hline $\begin{array}{l}\text { Realización de actividades en clase con } \\
\text { posterior entrega }\end{array}$ & 16 & 6,7 & 29 & 12,1 & 33 & 13,8 \\
\hline $\begin{array}{l}\text { Trabajos en clase y luego participar } \\
\text { activamente de las puestas en común }\end{array}$ & 76 & 31,7 & 34 & 14,2 & 33 & 13,8 \\
\hline Total & 240 & 100 & 240 & 100 & 240 & 100 \\
\hline
\end{tabular}

Cuando la información fue obtenida de forma cualitativa (cuestionarios abiertos y entrevistas), las respuestas de estudiantes y docentes se vincularon, principalmente, con los siguientes ejes (gráfico 1):

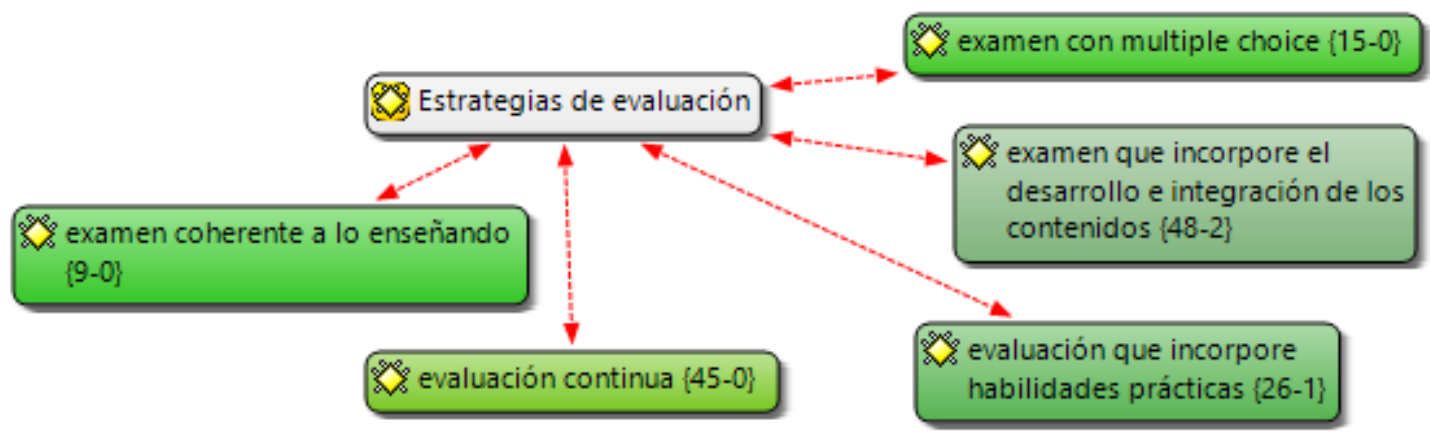

Gráfico 1. Estrategias de evaluación. Categoría y subcategorías. (Elaboración propia. Gráfico obtenido mediante el programa estadístico Atlas.ti).

Un aspecto que ha surgido de los estudiantes es la necesidad de que exista una coherencia entre los contenidos que se enseñan y lo que se evalúa:

"En cuanto a la evaluación, no veo ninguna complicación, siempre y cuando se tome lo pactado y no cosas que no se hayan visto en clase (multiple choice)" (estudiante 7). 
"Los contenidos deben ser correspondidos, en humana nos toman contenidos de aplicada y se dejan de lado cuestiones importantes de la fisiología” (estudiante 20).

"Que se pregunte como se dio en clase y sin rebuscar las preguntas o sin utilizar un lenguaje en el que no se habla en clase" (estudiante 24).

"preguntas consisas que hayan dado en clase y no preguntas que no tengan que ver con la cursada, ni grados superiores" (estudiante 34).

"Me gustaría que enseñen de alguna forma que el final no sea algo tan distinto a la cursada. (...) Me gustaría que me evaluen con los contenidos dados en clase" (estudiante 36).

"La manera que me gustó como me evaluaron fue en la que un profesor nos dio 80 preguntas un día antes y en el parcial tomaba 15 de esas 80 . Es buena esa metodología ya que te guía en lo que hay que estudiar" (estudiante 67).

"en su práctico dá 80 preguntas para contestar y en el parcial elije de esas preguntas" (estudiante 107).

"De una forma más articulada entre temas, ya que al fin es lo que toman en el final" (estudiante 109).

Luego, las opiniones rondaron en torno a "el examen multiple choice", para expresar su conformidad o desconformidad en torno a éste, o para solicitar que se lo complemente con otros tipos o diseños de examen. Se opinó acerca de la posibilidad de desarrollar, integrar contenidos y de incorporar el desenvolvimiento en habilidades prácticas en las evaluaciones. Con respecto al tema de "evaluación continua", hubo varios votos que apoyaron una continua y diversa cantidad de instancias de evaluación en el transcurso de la cursada de la materia. A continuación se describen tales ejes:

- $\quad$ Evaluación que incorpore el desarrollo e integración de los contenidos versus examen múltiple choice

- $\quad$ Evaluación que incorpore el desarrollo de habilidades prácticas

- $\quad$ Evaluación continua

Evaluación que incorpore el desarrollo e integración de los contenidos versus examen múltiple choice

Prevalece una constante referencia de los estudiantes hacia los exámenes de multiple choice. Pareciera ser que es un tipo de examen que se utiliza con frecuencia en estas materias. También se acude, en gran medida, al examen que posibilita el desarrollo de conceptos. Principalmente, se ha obtenido que:

- Los estudiantes que prefieren la estrategia evaluativa de múltiple choice no explicaron por qué, excepto en un caso donde se dijo que facilita la rápida corrección.

- Los estudiantes que rechazan el múltiple choice expresaron que por medio de este examen no pueden explicar sus respuestas ni expresar lo estudiado, tampoco les permite relacionar conceptos, y además, facilita la posibilidad de hacer trampa o zafar, los enunciados provocan confusión, generando incertidumbre sobre su resolución.

Los estudiantes prefieren un examen que combine diferentes tipos de preguntas, pudiendo en un mismo examen, explicar, desarrollar y relacionar conceptos, así como responder algunas preguntas más concretas al estilo múltiple opción o verdadero y falso. 
- Los estudiantes prefieren exámenes donde haya que desarrollar e integrar contenidos, se priorice el entendimiento sobre la memorización, y se aplique la teoría a posibles situaciones de la vida real y/o profesional.

- En el marco del ítem anterior, los estudiantes desean preparar, también, producciones escritas y, en menor medida, orales, como por ejemplo, realizar monografías, trabajos de investigación, trabajos prácticos y exposiciones orales.

Los docentes han desarrollado diferentes propuestas:

- Examen a libro abierto: el examen debería ser resolver, de forma escrita en el horario de clase, alguna posible situación de la futura vida laboral profesional, pudiendo consultar sus libros y con algún compañero, ya que es de la forma en que van a trabajar una vez recibidos.

Examen escrito a desarrollar: debería ser de esta forma, ya que permite relacionar los contenidos de diferentes unidades, también favorece la producción de escritura académica. Dificultad: el tiempo de corrección.

- Examen oral: según algunos, el examen debería ser oral porque permite que el estudiante explique sus respuestas, desarrolle sus ideas, pero no hay posibilidad porque se dispone de poco tiempo para evaluar y son muchos los estudiantes. Para otros, el examen oral no es el ideal porque juegan otros factores, principalmente, los nervios.

Examen múltiple choice: es el que se utiliza por su practicidad y rápida corrección, ya que el estudiante no debe redactar, sino marcar una opción. No asegura que el estudiante haya entendido la materia. Representa un número en un papel, de esta forma se tiene poco conocimiento sobre el proceso de aprendizaje que está realizando cada estudiante.

- $\quad$ Evaluación diversa: se debería evaluar de forma diversa, utilizando distintas técnicas y estrategias, debido a la complejidad que representa evaluar en el siguiente sentido: “¿cómo diseño actividades que me permitan evaluar lo que quiero que el alumno sepa hacer o sepa?" (docente 8/174).

\section{Evaluación que incorpore el desarrollo de habilidades prácticas}

La mayoría de los estudiantes que ha opinado al respecto, desea que:

Los exámenes tengan una parte teórica y una parte práctica, y que la parte teórica exija la aplicación de los contenidos a situaciones prácticas.

En el examen escrito se plantee una situación de la práctica, la cual ellos podrían llegar a enfrentarse en un futuro, tengan que analizarla, resolverla y explicar por qué se tomaron esas decisiones, basándose en la fisiología.

- $\quad$ En la parte práctica haya que realizar habilidades operativas, como ejecutar ciertos test, tomar la presión, reconocer la intensidad de diferentes esfuerzos físicos en distintas personas, aplicar lo aprendido entre compañeros, entre otros ejemplos, es decir, desenvolverse en situaciones que puedan darse en la futura práctica profesional.

Se integren teoría y práctica, por ejemplo, en proyectos de investigación, donde se estudie un tema y luego deba hacerse un trabajo de campo que implique el desarrollo de habilidades operativas.

Un solo docente ha opinado al respecto, su perspectiva es: 
- Utilizar más de una herramienta para evaluar, el examen debería componerse por una parte práctica, una parte teórica y una parte de resolución de problemas. Por ejemplo, incluiría lo práctico y la resolución de problemas preguntar por qué puede haberse desmayado una persona en una maratón, y lo teórico saber describir conceptos.

\section{Evaluación continua}

Los estudiantes valoran que:

Haya que hacer trabajos prácticos o traer preguntas respondidas a cada clase, tener guías de preguntas sobre los contenidos que se van enseñando, realizar monografías, planificaciones, trabajos de investigación, exposiciones orales, hacer evaluaciones o responder de forma oral preguntas todas las semanas/clases, y que se evalúe también la participación y la asistencia, además del parcial al final de la cursada. Los estudiantes desean que a estas actividades el docente les adjudique una nota, con el fin de que aporten al promedio en la nota final y para aprender de los errores que se puedan haber cometido. Esta dinámica estimula la lectura, favorece la constancia para llevar al día la materia y potencia el entendimiento y la comprensión de los contenidos, ya que, al evaluar de forma periódica y realizando un seguimiento sobre ella, tanto docente como estudiantes, pueden reconocer qué temas o aspectos no fueron comprendidos y hacer hincapié en ello. En este sentido, rendir un parcial al final del ciclo, como única instancia evaluativa, puede llevar a que el estudio de la materia se base, solamente, en obtener una nota.

Para los docentes:

Los estudiantes que cursan la materia por promoción sin examen final, tienen mayores requerimientos en cuanto tiempo destinado e instancias evaluativas, que aquellos que cursan la materia de forma regular. Existe diferencia en cuanto al proceso de aprendizaje, a la información que manejan y, en general, a la formación alcanzada, por estas diferentes vías de cursada, logrando una mayor formación quienes cursan por promoción sin examen final.

La evaluación continua permite conocer, en mayor medida, acerca del proceso de aprendizaje que realizan los estudiantes, en comparación con la evaluación de tipo transversal, la cual puede verse influenciada por diferentes inconvenientes.

Dificultades para que la evaluación sea continua: el tiempo que implica la corrección de trabajos, lo cual se encuentra aumentado por las falencias de los estudiantes para expresarse de forma escrita, en la redacción, la ortografía, entre otros, y lo mismo ocurre de forma oral, cuando lo exámenes requieren que el estudiante explique oralmente algún tema.

Experiencias favorables en el aula: organización en grupos para responder preguntas que luego debían exponerse al resto de la clase, teniendo como estímulo la competencia, es decir, el grupo que más sabía, sumaba más puntos.

\section{Discusión y conclusiones}

En consonancia con los resultados obtenidos, en el estudio de Gómez Ruiz y Quesada Sierra (2017), las principales valoraciones de los estudiantes sobre la aplicación de la evaluación formativa, fueron: la retroalimentación, la posibilidad de reflexionar, ser conscientes y aprender de los errores, y el hecho de aprender a evaluar. También se ha hallado una similitud en cuanto a la dificultad que encuentran los docentes en la evaluación continua en torno al 
tiempo que supone corregir los trabajos que realizan los estudiantes (Castejón Oliva et al., (2011), Gómez-Ruiz, Rodríguez-Gómez e Ibarra-Sáiz, 2013; Rodríguez Conde, 2005).

Otro aspecto que concuerda con los hallazgos alcanzados fue obtenido por Ricoy y Fernández-Rodríguez (2013), quienes observaron que el sistema de evaluación final favorece la adquisición de aprendizajes superficiales y puntuales de los contenidos de la asignatura que se desvanecen pronto finaliza la asignatura, por lo que, la evaluación única y al final del ciclo, debería empezar a perder valor y tender a su desaparición. En contraposición con ello, debería potenciarse el desarrollo de procedimientos complejos a través de técnicas e instrumentos de evaluación diversos, los cuales estarán en función de los objetivos que se persigan. Gessa Perera (2011) y López Pastor (2006), encontraron que las estrategias de evaluación de coevaluación o evaluación conjunta profesor-alumno constituyen una oportunidad para motivar, mejorar y consolidar el aprendizaje.

Al igual que en estudios recientes (López Pastor, 2006; Padilla Carmona y Gil Flores, 2008; Davini, 2008; Fraile Aranda, 2009; Castejón Oliva et al., 2011; Gómez Ruiz y Quesada Sierra, 2017)., la experiencia de estudiantes y docentes indica que la evaluación debería ser formativa, orientada hacia el aprendizaje y, por tanto, continua, formando parte de todo el proceso de aprendizaje y valorándose diferentes tipos de aprendizajes y competencias, útiles para la futura práctica profesional y para la vida personal.

En este estudio, las opiniones y preferencias de estudiantes y docentes estuvieron vinculadas a la evaluación que incorpore el desarrollo e integración de los contenidos versus examen múltiple choice, la evaluación que incorpore el desarrollo de habilidades prácticas, y la evaluación continua.

Hubo una extensa cantidad de opiniones de los estudiantes sobre los exámenes escritos múltiple choice o a desarrollar, concluyéndose que es la forma de evaluar que se utiliza con más frecuencia en estas asignaturas. Se conoció que los estudiantes prefieren que se los evalúe por su trabajo y participación en clase. Se destacó el pedido de los estudiantes para que se incorpore la evaluación de habilidades prácticas en los exámenes, y que se evalúe de forma continua.

Los estudiantes, en general, valoran poder explicar, desarrollar e integrar contenidos, pudiendo incorporarse, también, algunas preguntas concretas y problemas a resolver que vinculen el contenido con la práctica. Si bien algunos estudiantes están de acuerdo con el examen múltiple choice, muchos han expresado que provoca confusión, favorece la trampa y no permite explicar lo estudiado. Otras opciones de examen pueden ser la elaboración de monografías, trabajos de investigación y exposiciones orales, si es posible que integren la teoría a la práctica profesional. Se ha hecho énfasis en la incorporación de una parte práctica en los exámenes, donde por ejemplo haya que resolver un problema ligado a la labor profesional basándose en los conocimientos sobre la fisiología, y la incorporación de actividades corporales que se involucren en el desarrollo de habilidades operativas vinculadas a la futura profesión. Los estudiantes quieren ser evaluados a partir de la realización de actividades todas las semanas, como trabajos prácticos y responder preguntas de forma escrita u oral, que otorguen nota, con el fin de aprender de los errores y que se promedie con la nota final, además, las actividades que se implementan de forma continua favorecen la constancia, la lectura, el estar al día, lo que a su vez mejora la comprensión de los contenidos.

Sobre este tópico, los resultados permiten afirmar que no existe una preferencia sobre un tipo de evaluación, sino una diversidad de experiencias y valoraciones. Los docentes entienden al examen escrito a desarrollar como una buena opción ya que permite relacionar los contenidos y producir académicamente, además, en este tipo de examen se pueden 
incorporar problemas a resolver; al examen múltiple choice como una opción práctica, de fácil corrección, pero limitada para conocer si el estudiante comprende la materia; al examen oral como aquel que permite a los estudiantes expresarse, pero lo perjudica la situación de tensión y nervios; al examen a libro abierto como una opción que posibilita la resolución de algún problema práctico utilizando los libros como soporte. Los docentes notan que los estudiantes que eligen cursar por promoción sin examen final, alcanzan una mejor formación que quienes no lo hacen; esto se debe a los mayores requerimientos en cuanto a carga horaria, actividades y exámenes a resolver, además, evaluar de forma continua por medio de distintas actividades, permite conocer mejor el proceso de aprendizaje de los estudiantes.

Por tanto, las estrategias de evaluación formativas, orientadas hacia el aprendizaje, y por tanto continuas, son las más apreciadas por los docentes y estudiantes, ya que les posibilitan la adquisición de aprendizajes no sólo para la práctica profesional sino para la vida en general.

\section{Referencias}

Berbén, A. B. G., de la Fuente, J., Benítez, J. L. y Fernández Cabezas, M. (2007). Análisis de las preferencias de enseñanza del profesorado en formación. III Congreso Internacional de Formación del Profesorado. En: Romero López, M. A., Gámiz Sánchez, V. A. y Crisol Moya, E. (Coords.). III Congreso Internacional de Formación del Profesorado. Innovación, Formación y Profesionalización educativa. Recuperado de https://www.researchgate.net/profile/Francisco_Alcaide/publication/283321476_Mo delos_para_la_formacion_inicial_del_profesorado_de_Secundaria_segun_los_aspir antes_de_Ciencias/links/5633486608ae242468db8656/Modelos-para-la-formacioninicial-del-profesorado-de-Secundaria-segun-los-aspirantes-de-Ciencias.pdf.

Castejón Oliva, F. J., López-Pastor, V. M., Julián Clemente, J. A. y Zaragoza Casterad, J. (2011). Evaluación formativa y rendimiento académico en la formación inicial del profesorado de Educación Física. Revista Internacional de Medicina y Ciencias de la Actividad Física y del Deporte, 11(42), 238-346. Recuperado de http://www.redalyc.org/pdf/542/54222171007.pdf.

Colina Colina, L. (2007). La investigación en la educación superior y su aplicabilidad social. Laurus, $\quad$ 13(25), 330-353. Recuperado de http://www.redalyc.org/pdf/761/76111479016.pdf.

Córdoba Gómez, F. J. (2006). La evaluación de los estudiantes: una discusión abierta. Revista Iberoamericana de Educación, 39(7), 1-8.

Davini, M. C. (2008). Métodos de enseñanza. Didáctica general para maestros y profesores. Buenos Aires, Argentina: Santillana. Recuperado de https://practicasdelaen2.files.wordpress.com/2013/04/mc3agtodos-deenseczb1anza-davini.pdf.

Fraile Aranda, A. (2009). La enseñanza superior: Un controvertido camino para el profesorado universitario desde la convergencia europea. Universidades, (40), 3-17. Recuperado de http://www.redalyc.org/html/373/37313028002/.

Gessa Perera, A. (2011). La coevaluación como metodología complementaria de la evaluación del aprendizaje. Análisis y reflexión en las aulas universitarias. Revista de Educación, 354, 749-764. Recuperado de http://www.revistaeducacion.educacion.es/re354/re354_30.pdf. 
Gómez Ruiz, M. A. y Quesada Serra, V. (2017). Coevaluación o Evaluación Compartida en el Contexto Universitario: La Percepción del Alumnado de Primer Curso. Revista Iberoamericana de Evaluación Educativa, 10(2), 9-30. Recuperado de https://revistas.uam.es/index.php/riee/article/view/8153.

Gómez-Ruiz, M. A., Rodríguez-Gómez, G. e Ibarra-Sáiz, M. S. (2013). Desarrollo de las competencias básicas de los estudiantes de Educación Superior mediante la eEvaluación orientada al aprendizaje. RELIEVE, 19(1), 1-17. Recuperado de https://ojs.uv.es/index.php/RELIEVE/article/view/2457.

Hernández Pina, F., Arán Jara, A. y Salmerón Pérez, H. (2012). Enfoques de aprendizaje y metodologías de enseñanza en la universidad. Revista Iberoamericana de Educación, 60(3), 1-12. Recuperado de file:///C:/Users/s/Downloads/4878Hdez\%20(1).pdf.

Hernández Sampieri, R. H., Fernández Collado, C. y Baptista Lucio, P. (2006). Metodología de la investigación. Recuperado de http://files.especializaciontig.webnode.com/200000775-097910b6co/sampieri-et-al-metodologia-de-lainvestigacion-4ta-edicion-sampieri-2006_ocr.pdf.

Hernández Sampieri, R., Fernández Collado, C. y Baptista Lucio, M. (2014). Metodología de la investigación. (6a edición). México D. F: McGraw-Hill. Recuperado de http://observatorio.epacartagena.gov.co/wp-content/uploads/2017/08/metodologiade-la-investigacion-sexta-edicion.compressed.pdf

López Pastor, V. M. (2006). El papel de la evaluación formativa en el proceso de convergencia hacia el E.E.E.S. Análisis del estado de la cuestión y presentación de un sistema de intervención. Revista Interuniversitaria de Formación del Profesorado, 20(3), 93-119. Recuperado de http://www.redalyc.org/articulo.oa?id=27411311005.

Moreno Olivos, T. (2009). La evaluación del aprendizaje en la universidad. Tensiones, contradicciones y desafíos. RMIE, 14(41), 563-591. Recuperado de http://www.scielo.org.mx/scielo.php?script=sci_arttext\&pid=S140566662009000200010 .

Padilla Carmona, T. y Gil Flores, J. (2008). La evaluación orientada al aprendizaje en la Educación Superior: condiciones y estrategias para su aplicación en la docencia universitaria. Revista española de pedagogía, 66(241), 467-486. Recuperado de file:///C:/Users/s/Downloads/Dialnet-

LaEvaluacionOrientadaAIAprendizajeEnLaEducacionSup-2709011.pdf.

Pavié, A. (2011). Formación docente: hacia una definición del concepto de competencia profesional docente. REIFOP, 14(1), 67-80. Recuperado de file://C:/Users/s/Downloads/Dialnet-FormacionDocente-3678767\%20(1).pdf.

Ricoy, M. C. y Fernández-Rodríguez, J. (2013). La percepción que tienen los estudiantes universitarios sobre la evaluación: un estudio de caso. Educación XX1, 16(2), 321-341. Recuperado de http://www.redalyc.org/pdf/706/70626451006.pdf.

Rodríguez Conde, M. J. (2005). Aplicación de las TIC a la evaluación de alumnos universitarios. Teoría de la Educación. Educación y Cultura en la Sociedad de la Información, 6(2), 1-17. Recuperado de http://www.redalyc.org/articulo.oa?id=201021055002.

Sander, P., Stevenson, K., King, M. y Coates, D. (2000) University Students' Expectations of Teaching. Studies in Higher Education, 25(3), 309-323. Doi: $10.1080 / 03075070050193433$. 